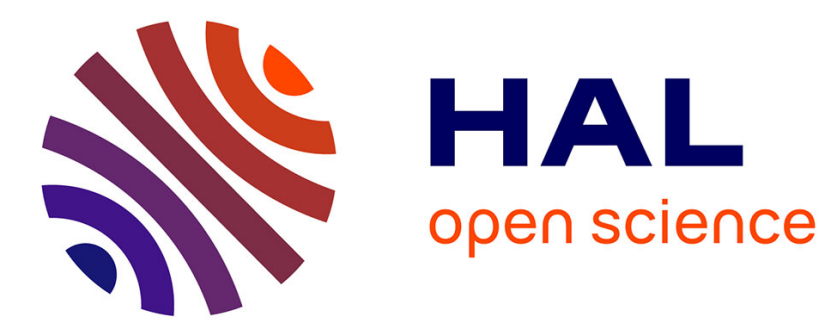

\title{
Noise behavior of Spline Mojette FBP reconstruction
}

\author{
Myriam Servières, Nicolas Normand, Yves Bizais, Jean-Pierre Guédon
}

\section{To cite this version:}

Myriam Servières, Nicolas Normand, Yves Bizais, Jean-Pierre Guédon. Noise behavior of Spline Mojette FBP reconstruction. SPIE Medical Imaging 2005: Image Processing, Feb 2005, San Diego, CA, United States. pp.2100-2109, 10.1117/12.593390 . hal-01500579

\section{HAL Id: hal-01500579 \\ https://hal.science/hal-01500579}

Submitted on 16 May 2017

HAL is a multi-disciplinary open access archive for the deposit and dissemination of scientific research documents, whether they are published or not. The documents may come from teaching and research institutions in France or abroad, or from public or private research centers.
L'archive ouverte pluridisciplinaire HAL, est destinée au dépôt et à la diffusion de documents scientifiques de niveau recherche, publiés ou non, émanant des établissements d'enseignement et de recherche français ou étrangers, des laboratoires publics ou privés. 


\title{
Noise behavior of Spline Mojette FBP reconstruction
}

\author{
Myriam Servières $^{a}$, Nicolas Normand ${ }^{a}$, \\ Yves Bizais $^{b}$ and JeanPierre Guédon ${ }^{a}$ \\ ${ }^{a}$ IRCCyN/IVC - UMR CNRS 6597, Image \& Video Communication, \\ École polytechnique de l'Université de Nantes, \\ La Chantrerie, BP 50609, 44306 Nantes Cedex 3 \\ ${ }^{b}$ Laboratoire de Traitement de l'Information Médicale, \\ Bâtiment 2bis (I3S) CHU Morvan - 5, Av. Foch, \\ 29609 Brest CEDEX - FRANCE
}

\begin{abstract}
The goal of this paper is to characterize the noise properties of a spline Filtered BackProjection (denoted as FBP) reconstruction scheme. More specifically, the paper focuses on angular and radial sampling of projection data and on assumed local properties of the function to be reconstructed. This new method is visually and quantitatively compared to standard sampling used for FBP scheme.

In the second section, we recall the sampling geometry adapted to the discrete geometry of the reconstructed image. Properties of the discrete zero order Spline Ramp filter for classic angles and discrete angles generated from Farey's series reconstruction are used to generate their equivalent representations for first order Spline filters. Digital phantoms are used to assess the results and the correctness of the linearity and shift-invariantness assumption for the discrete reconstructions. The filter gain has been studied in the Mojette case since the number of projections can be very different from one angle to another.

In the third section, we describe the Spline filter implementation and the continuous/discrete correspondence.

In section 4, Poisson noise is added to noise-free onto the projections. The reconstructions between classic angle distribution and Mojette acquisition geometry are compared. Even if the number of bins per projections is fixed for classic FBP while it varies for the Mojette geometry (leading to very different noise behavior per bin) the results of both algorithms are very close.

The discussion allows for a general comparison between classic FBP reconstruction and Mojette FBP. The very encouraging results obtained for the Mojette case conclude for the developments of future acquisition devices modeled with the Mojette geometry.
\end{abstract}

\section{INTRODUCTION}

Sampling is still a major issue for tomographic devices. The last decade has seen breakthroughs as fully 3D CT that allows for new and better device sampling. New acquisition devices have to be re-understood via the best way the data can be computed thereafter. For us, the sooner the data fill a proper projection space, the more precise the final tomographic reconstruction because no compromise has to be done with noisy data inside the tomographic box. This was the starting point for deriving the Mojette transform ten years ago. This version of a discrete exact Radon transform that can only (at the beginning) reconstruct exact data was extended. Last year, we presented a

\footnotetext{
Further author information: (Send correspondence to Myriam Servières)

$a$ Email: \{myriam.servieres, nicolas.normand, jean-pierre.guedon \}@polytech.univ-nantes.fr

Phone: + 332406830 48, Fax: + 33240683232

${ }^{b}$ Email: Yves.Bizais@univ-brest.fr
} 
revisited version of the FBP algorithm ${ }^{6}$ using the sampling point of view of the Mojette transform and results are recalled in section 2 . The pixel intensity distribution model used for the spline 0 also called the Haar function box (low frequency part of the Haar wavelet). In this paper, we start by extending this previous result to spline of higher degrees. From such a filter, we can implement a set of filters of spline of order $n$ depending either to the spline of order 0 or 1 . These filter implementations are described in section 3. Moreover, these filters respect the general sampling theorem of UnserAlDroubi ${ }^{1}$ because of the structure of the initial data sampling. It must be pointed out that it was not "exactly" the case for the initial implementation of Guedon-Bizais ${ }^{4}$ for spline filters where they used an oversampling ratio between projection sampling path and pixel resolution to overcome the structuring problem of the sampling that is naturally solve with the Mojette geometry.

Our goal in this paper is to understand the noise characteristics for the Mojette tomographic operator. Of course its Fourier components are very different from a standard FBP operator because of the projection sampling that implies the 2D sampling in the Fourier domain to match a cartesian grid instead of the classical radial one. Section 4 presents the results of very simple phantoms designed for exact and noisy tomographic reconstructions both for standard and Mojette sampling. Section 5 discusses these results in terms of how the comparison can be done. This firstly adresses the way of distributing the noise among the recorded bins and secondly the simple figure of merit used here.

\section{FBP MOJETTE}

\subsection{Mojette sampling geometry}

The Mojette projector is a linear discrete exact version of the Radon transform. To obtain this projector, the object is discretized using a pixel model, as described in the next section, and the angular sampling is performed as follows.

The projection directions are defined by $\tan \theta=q / p$ with $p$ relatively $q$ prime and $q>0$ (except for $q=0, p=1)$. The sampling path on the projection is $t=\frac{\Delta b}{\sqrt{p^{2}+q^{2}}}$ where $\Delta$ is the pixel size and $b$ the bin indice. This sampling gives an exact backprojection with no interpolation onto the projection..$^{5}$ The sampling geometry is adapted to the discrete geometry of the digital reconstructed image.

The Mojette FBP transform allows one to do tomographic reconstruction with good accuracy compared to the classical reconstruction method, especially on the shape boundaries. Moreover, the FBP scheme gives a complete continuous-discrete equivalence ${ }^{6}$ due to the discretization scheme.

\subsection{Use a pixel model in projection modeling}

To map the object onto the discrete space, we use a pixel model. The choice of a pixel model will define the Mojette projector.

The Mojette projector depends on the pixel model $p m(x, y)$ chosen to describe the object $f(x, y)^{2}$ :

$$
f(x, y)=\sum_{k=-\infty}^{+\infty} \sum_{l=-\infty}^{+\infty} f(k, l) p m(x-k, y-l)
$$

and defines the general Mojette projection:

$$
\operatorname{proj}_{p m}(b, p, q)=\sum_{k=-\infty}^{+\infty} \sum_{l=-\infty}^{+\infty} f(k, l) \int_{-\infty}^{+\infty} \int_{-\infty}^{+\infty} p m(x-k, y-l) \delta(b+q x-p y) d x d y .
$$

With such an expression, and a Dirac pixel model:

$$
p m(x, y)=\delta(x) \delta(y),
$$


we define the Dirac Mojette projector as:

$$
\operatorname{proj}_{\delta}(b, p, q)=\sum_{k=-\infty}^{+\infty} \sum_{l=-\infty}^{+\infty} f(k, l) \Delta(b+q k-p l) .
$$

The use of this projector with the discrete angle is illustrated (Fig. 1).

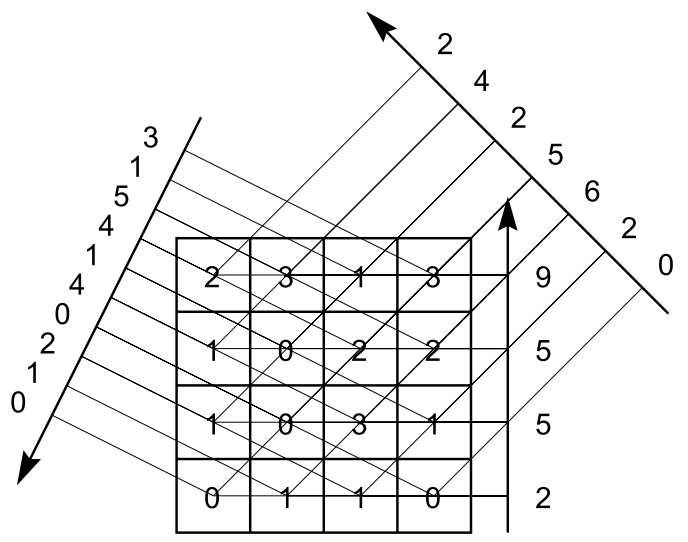

(a) Dirac Mojette projection

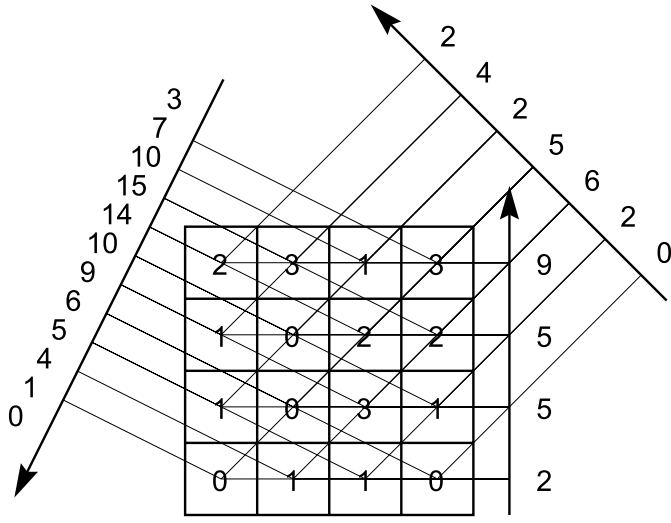

(b) Spline 0 Mojette projection (with a not normalized trapezoid)

Figure 1. Mojette projections of a $4 \times 4$ image with projections directions $(p, q) \in\{(1,0),(1,1),(-2,1)\}$

With the same definition, the Spline Mojette projector is define at any Spline order n:

$$
\operatorname{proj}_{\beta_{n}}(b, p, q)=\sum_{k=-\infty}^{+\infty} \sum_{l=-\infty}^{+\infty} f(k, l) \int_{-\infty}^{+\infty} \int_{-\infty}^{+\infty} \beta_{n}(x-k, y-l) \delta(b+q x-p y) d x d y .
$$

In this paper, we will use only Spline $0, \beta_{0}(t)=\left\{\begin{array}{l}1 \text { if }|t|<\frac{1}{2} \\ 1 \text { if }|t|=\frac{1}{2} \\ 0 \text { elsewhere }\end{array}\right.$, and Spline 1 pixel model $\beta_{1}(t)=$ $\beta_{0}(t) \star \beta_{0}(t)$ where $\star$ denotes the convolution operator.

\subsection{Mojette backprojection}

The Mojette backprojector is defined as the dual of the Mojette projector. For a Dirac pixel model, the Mojette exact backprojector is defined $a^{5}$ :

$$
\left[M^{*} \operatorname{proj}\right](k, l)=\sum_{i=-\infty}^{+\infty} \sum_{j=-\infty}^{+\infty} \delta(k-i) \delta(l-j) \sum_{b=-\infty}^{+\infty} \operatorname{proj}(b, p, q) \Delta(b+q i-p j)
$$

This means for one projection $(p, q): f_{p, q}(k, l)=M_{\delta}^{*}$ proj The Mojette dual operator $M^{*}$ corresponds to an exact discrete backprojector operator. Then, using a Mojette angle sampling allows to backproject onto pixel centers without any interpolation. The angular assessment of the Mojette FBP scheme has been done with Mojette angles equally sampled given by larges Farey series. 
The Farey series of order $N\left(F_{N}\right)$ is the set of all fractions in lowest terms between 0 and 1 whose denominators do not exceed $N$, arranged in order of magnitude. For example, $F_{4}$ is composed of $\left\{\frac{0}{1}, \frac{1}{4}, \frac{1}{3}, \frac{1}{2}, \frac{2}{3}, \frac{3}{4}, \frac{1}{1}\right\}$. Using symmetry, we found discrete angles into the whole space with the fraction denominator as $p$ and the numerator as $q$. For a square image $N \times N$, using the Farey's series of order N, all the discrete directions to go from a pixel to an other one in the image are obtained. Our test images are $128 \times 128$, so we first generate $20088\left(\operatorname{Card}\left(F_{128}\right)\right)$ projections and in this set we choose equally distributed discrete angles that give a good reconstruction. ${ }^{7}$

\section{FILTER IMPLEMENTATION AND CONTINUOUS DISCRETE CORRESPONDENCE}

\subsection{Spline filter definition}

\subsubsection{Ramp filter for Spline of order 0: $k_{0}$}

The Spline 0 Mojette operator that corresponds to model the bin value by the entire continuous line summation (not the discrete line as the Dirac Mojette operator) will be used. The continuous exact Spline 0 FBP filter was derived in Guédon and Bizais ${ }^{4}$ and its expression in the Fourier domain is given by:

$$
K_{0}(\nu, \theta)=\Delta^{2} \pi|\nu| \operatorname{sinc}(\nu \cos \theta) \operatorname{sinc}(\nu \sin \theta),
$$

where the apodisation function corresponds to the projection of the filter onto the Spline space of order 0 . The pixel size is $\Delta$.

The inverse fourier transform of (Eq.7) was derived as

$$
k_{0}(t, \theta)=\frac{1}{\pi \sin (2 \theta)} \ln \left|\frac{t^{2}-\frac{\Delta^{2}}{4}(1+\sin (2 \theta))}{t^{2}-\frac{\Delta^{2}}{4}(1-\sin (2 \theta))}\right|,
$$

for $t \neq 0$ and $\left.\theta \in] 0, \frac{\pi}{4}\right]$ and $k_{0}(t, 0)=\frac{-1}{\pi} \frac{2 \Delta}{4 t^{2}-1}$.

Discretizing with Mojette angles $\left(\tan \theta=\frac{q}{p}\right)$ with the projection sampling $t=\frac{b \Delta}{\sqrt{p^{2}+q^{2}}}$ leads to:

$$
k_{0}(b, p, q)=\frac{p^{2}+q^{2}}{2 \pi p q} \ln \left|\frac{b^{2}-\left(\frac{p+q}{2}\right)^{2}}{b^{2}-\left(\frac{p-q}{2}\right)^{2}}\right|,
$$

for $b \neq 0$ and $(p, q) \neq(1,0)$ and $k_{0}(b, 1,0)=\frac{-1}{\pi} \frac{2 \Delta}{4 b^{2} \Delta^{2}-1}$.

This filter can not be implemented in a straightforward manner in the spatial domain. It exhibits discontinuities at the points where the projection of the pixel (described by a trapezoidal shape) is continuous but not differentiable. The Ramp filter acting as a derivative operator, the values at these points have to be computed using a Dirichlet condition. Implementing the FBP Mojette operator uses the Mojette projector, the $k_{0}$ filter and the Mojette backprojector. This FBP Mojette scheme is exact for the filter when an infinite number of angles is used and exact for the backprojection of these filtered angles.

\subsubsection{Ramp filter for Spline of order 1: $k_{1}$}

The properties of the $k_{0}(t, \theta)$ (respectively $k_{0}(b, p, q)$ ) discrete Spline 0 Ramp filter for classic angles (resp. discrete angles derived from Farey's series with $\tan \theta=q / p$ ) reconstruction are used to generate their equivalent representations for first order Spline filters $k_{1}(t, \theta)$ and $k_{1}(b, p, q)$ :

$$
K_{1}(\nu, \theta)=K_{0}(\nu, \theta) \times W_{0}(\nu, \theta)
$$


where $K_{0}(\nu, \theta)=\pi \Delta^{2}|\nu| \operatorname{sinc}(\Delta \nu \cos \theta) \operatorname{sinc}(\Delta \nu \sin \theta)$ and $W_{0}(\nu, \theta)$ is the Fourier transform of the projection of the Spline 0 pixel model in the $\theta$ direction. The $k_{1}$ filter is obtained in the spatial domain for the continuous case

$$
k_{1}(t, \theta)=\frac{1}{2 \pi \sin ^{2} 2 \theta} \times\left[\begin{array}{l}
8 t^{2} \ln |t| \\
-4(t-\Delta \sin \theta)^{2} \ln |t-\Delta \sin \theta| \\
-4(t+\Delta \sin \theta)^{2} \ln |t+\Delta \sin \theta| \\
-4(t-\Delta \cos \theta)^{2} \ln |t-\Delta \cos \theta| \\
-4(t+\Delta \cos \theta)^{2} \ln |t+\Delta \cos \theta| \\
+2(t+\Delta \cos \theta+\Delta \sin \theta)^{2} \ln |t+\Delta \cos \theta+\Delta \sin \theta| \\
+2(t+\Delta \cos \theta-\Delta \sin \theta)^{2} \ln |t+\Delta \cos \theta-\Delta \sin \theta| \\
+2(t-\Delta \cos \theta+\Delta \sin \theta)^{2} \ln |t-\Delta \cos \theta+\Delta \sin \theta| \\
+2(t-\Delta \cos \theta-\Delta \sin \theta)^{2} \ln |t-\Delta \cos \theta-\Delta \sin \theta|
\end{array}\right],
$$

This corresponds for the Mojette case to:

$$
k_{1}(b, p, q)=\frac{\Delta^{2}\left(p^{2}+q^{2}\right)}{8 \pi p^{2} q^{2}} \times\left[\begin{array}{l}
8 b^{2} \ln |b| \\
-4(b-q)^{2} \ln |b-q| \\
-4(b+q)^{2} \ln |b+q| \\
-4(b-p)^{2} \ln |b-p| \\
-4(b+p)^{2} \ln |b+p| \\
+2(b+p+q)^{2} \ln |b+p+q| \\
+2(b+p-q)^{2} \ln |b+p-q| \\
+2(b-p+q)^{2} \ln |b-p+q| \\
+2(b-p-q)^{2} \ln |b-p-q|
\end{array}\right] .
$$

For knots as $b= \pm p, b= \pm q, b=0$ we use $\lim _{x \rightarrow 0} x^{2} \ln x \rightarrow 0$, and for the angle $(1,1)$ where there is still a singularity we compute an interpolation.

\subsection{Continuous-Discrete equivalence}

The use of the Spline 0 pixel model and the $k_{0}$ filter gives a continuous to discrete equivalence for all the FBP processes. ${ }^{6}$ The $K_{0}$ Fourier expression (Eq.7) is divided in two parts: a perfect Ramp filter and a trapezoidal shape. This trapezoid is the projection of the Spline 0 pixel Model. (Eq.5) with the Spline 0 pixel model represents the use of the corresponding Riesz basis (constructed by tensorial product of $\beta_{0}$ ) to get the continuous-discrete equivalence of the original image $f(x, y)$. Applying the FBP Mojette scheme, the $f(k, l)$ is projected using the Spline 0 Mojette projection. The projections are convolved with the $k_{0}$ filter, i.e. with the perfect Ramp filter then with a trapezoid. The last stage is the Dirac Mojette backprojection. These stages are equivalent to a Spline 0 Mojette projection, a filtering with the Ramp filter and a Spline 0 Mojette backprojection. For the Spline 0 $\beta_{0}=\stackrel{\circ}{\beta}_{0}$ for the Unser-Aldroubi scheme, where $\stackrel{\circ}{\beta}_{0}$ is the dual function of $\beta_{0}$.

This equivalence is generalized following the general sampling theory (fig.2). The same equivalence can be shown for all spline order.

$k_{n}$ reconstructions are implemented by:

- a Ramp filtering step

- followed by $n$ convolutions with $w_{0}$, (evntually no convolution for $k_{0}$ )

- a backprojection step.

The last two stages are equivalent to a Spline $n$ backprojection. With the dual function of the Spline interpolator, a continuous object equivalent to the discrete one reconstructed is obtained. 


\begin{tabular}{|c|c|c|c|c|c|c|c|c|c|c|}
\hline \multirow{4}{*}{$f(x, y) \in L_{2}$} & $\stackrel{* \beta_{0}}{\longrightarrow}$ & $f_{0}(k, l)$ & $\stackrel{M_{\delta}}{\longrightarrow}$ & $p_{0}(b, p, q)$ & $\stackrel{* k_{0}}{\longrightarrow}$ & $\tilde{p}_{0}(b, p, q)$ & $\stackrel{M_{S}^{*}}{\rightarrow}$ & $f_{0}(k, l)$ & $\stackrel{\stackrel{\circ}{\beta_{0}}}{\rightarrow}$ & $f_{0}(x, y)$ \\
\hline & $\stackrel{* \beta_{1}}{\longrightarrow}$ & $f_{1}(k, l)$ & $\stackrel{M_{\delta}}{\longrightarrow}$ & $p_{1}(b, p, q)$ & $\stackrel{* k_{1}}{\longrightarrow}$ & $\tilde{p}_{1}(b, p, q)$ & $\stackrel{M_{\delta}^{*}}{\longrightarrow}$ & $f_{1}(k, l)$ & $\stackrel{\beta_{1}}{\rightarrow}$ & $f_{1}(x, y)$ \\
\hline & $\vdots$ & & $\vdots$ & & $\vdots *_{n} w_{0}(b, p, q)$ & & $\vdots$ & & & \\
\hline & $\stackrel{* \beta_{n}}{\rightarrow}$ & $f_{n}(k, l)$ & $\stackrel{M_{\delta}}{\longrightarrow}$ & $p_{n}(b, p, q)$ & $\stackrel{* k_{n}}{\rightarrow}$ & $\tilde{p}_{n}(b, p, q)$ & $\stackrel{M_{S}^{*}}{\rightarrow}$ & $f_{n}(k, l)$ & $\stackrel{\stackrel{\circ}{\beta_{n}}}{\rightarrow}$ & $f_{n}(x, y)$ \\
\hline
\end{tabular}

Figure 2. Generalized continuous discrete equivalence using Spline pixel models and Spline based filters.

\section{NOISY RECONSTRUCTION}

To simulate a tomographic acquisition, the FBP Mojette reconstruction is performed with noisy data for a different number of projections but with the same total number of photons. If $T$ is the total photon numbers, $S$ the total sum of the pixels density and $I$ the number of projections, we have:

$$
a \times S \times I=T,
$$

where $a$ is a multiplicative factor. Before noise addition, the sum on each projection equals to $a S$.

The phantoms background is $\frac{1}{4}$. The first test image is a square $128 \times 128$ image with $17 \times 17$ centered pixels with a Dirichlet condition with the value 1 in the centered $15 \times 15$ square, $\frac{5}{8}$ in the boundaries and $\frac{7}{16}$ in the corners (fig.3a). The second one is a disk with 21 pixels diameters and the pixels value on the boundaries depends on intercepted surface between the pixel and the disk (fig.3b).

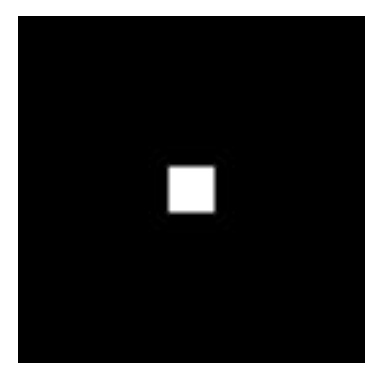

(a) square $128 \times 128$ phantom

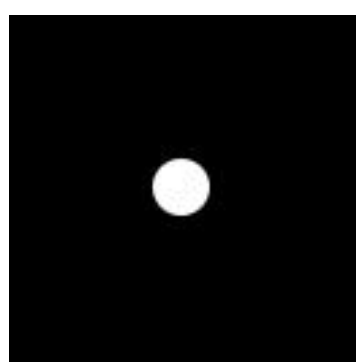

(b) disk $128 \times 128$ phantom

Figure 3. Test images

A Poisson noise is produced and added onto the projections corrected due to the number of bins per projection. The reconstructions are compared for classic angle distribution and Mojette acquisition geometry. Even if the number of bins per projections is fixed for classic FBP and varies for the Mojette geometry (leading to very different noise behavior per bin) the results of both algorithms are very close. All the reconstructed images are normalized between 0 and 1 . The FBP reconstruction are performed for 16, 32, 64, 128 and 256 projections. The FBPMojette and the classical reconstruction are first computed with the $k_{0}$ filter then with the $k_{1}$ one. The FBP Mojette scheme is a Spline 0 Mojette projection, a $k_{0}$ filtering stage then a Dirac Mojette backprojection, than it is a Spline 1 Mojette projection, a $k_{1}$ filtering stage, a Dirac Mojette backprojection and we obtain 
Table 1. Square image reconstruction, the resulting images are normalized onto [0,1]. (a) Spline 0 reconstruction with uniformly distributed Katz angles filtered by $k_{0}(b, p, q)$ and backprojected with a Mojette backprojection, (b) Classical reconstruction with regularly distributed angles filtered by $k_{0}(t, \theta)$ and backprojected with a classical backprojection, (c) Spline 1 reconstruction with uniformly distributed Katz angles filtered by $k_{1}(b, p, q)$ and backprojected with a Mojette backprojection, (d) Classical square image reconstruction with regularly distributed angles filtered by $k_{1}(t, \theta)$ and backprojected with a classical backprojection

(a)
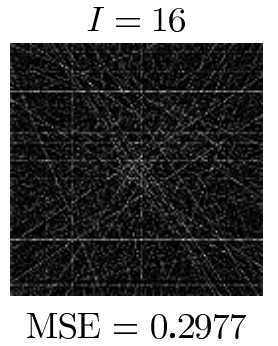

(b)

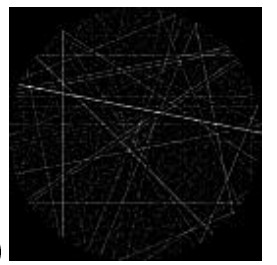

$\mathrm{MSE}=0.3560$

(c)

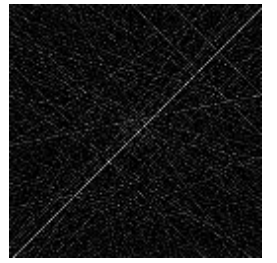

$\mathrm{MSE}=0.3373$

(d)

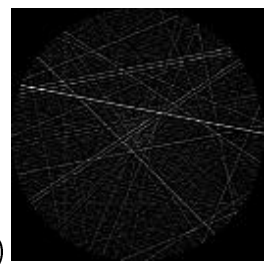

$\mathrm{MSE}=0.3280$
$I=32$

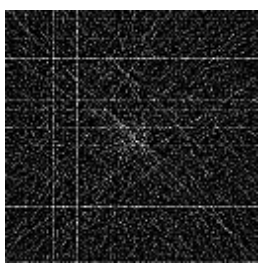

$\mathrm{MSE}=0.2711$

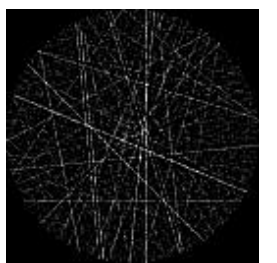

$\mathrm{MSE}=0.3110$

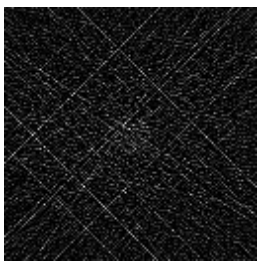

$\mathrm{MSE}=0.3075$

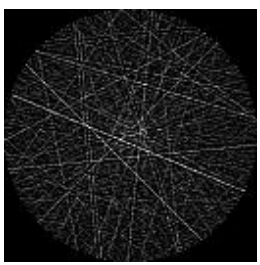

$\mathrm{MSE}=0.2672$
$I=64$

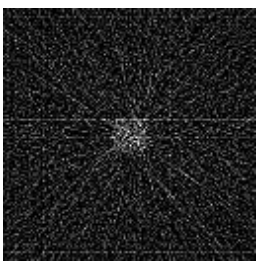

$\mathrm{MSE}=0.2203$

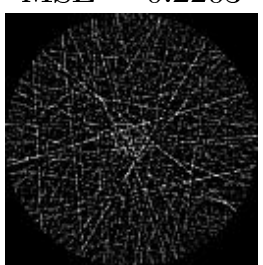

$\mathrm{MSE}=0.2797$

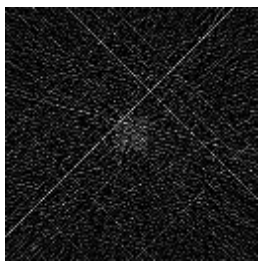

$\mathrm{MSE}=0.2773$

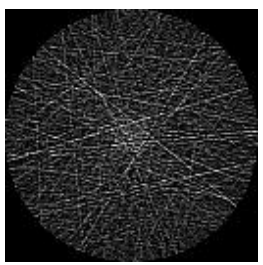

$\mathrm{MSE}=0.2193$
$I=128$

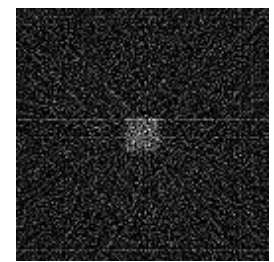

$\mathrm{MSE}=0.2035$

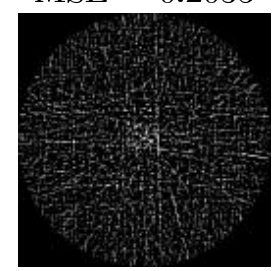

$\mathrm{MSE}=0.2718$

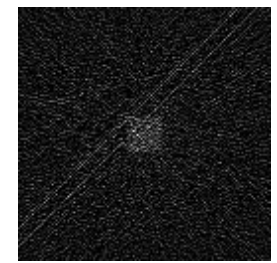

$\mathrm{MSE}=0.2600$

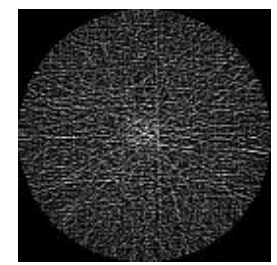

$\mathrm{MSE}=0.1932$
$\mathrm{I}=256$

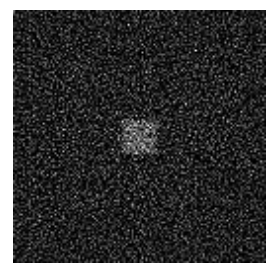

$\mathrm{MSE}=0.1867$

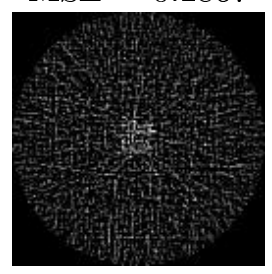

$\mathrm{MSE}=0.2472$

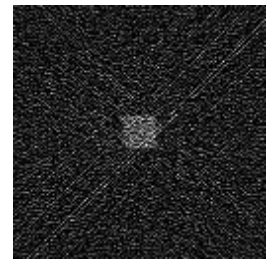

$\mathrm{MSE}=0.1978$

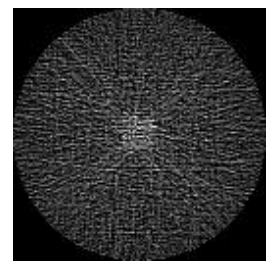

$\mathrm{MSE}=0.1762$

an image from the reconstructed coefficients with the Spline 1 dual function as explained before. The classic reconstruction is performed with 128 bins onto each projection. Here $T=1,000,000$ counts and $S=4312.75$ for the square phantom and $S=4355.77$ for the disk phantom.

The MSE is calculated onto the phantom shape on a $27 \times 27$ pixel square centered onto the square shape and on a $24 \times 24$ square centered onto the disk shape.

\section{DISCUSSION}

\subsection{Spline implementation}

The spline 0 Mojette filter corresponds to the differentiation of the trapezoidal shape made at discrete knots and for discrete angles that select every specific non differentiable location for $b=\frac{p+q}{2}$ and $b=\frac{p-q}{2}$. Because angles were assumed equally distributed with the previous spline implementation (i.e. $\left.\frac{\pi}{128}\right)^{4}$ these singularities were not computed at that time. For the spline 1 Mojette filter, the 
Table 2. round image reconstruction, the resulting images are normalized onto [0,1]. (a) Spline 0 reconstruction with uniformly distributed Katz angles filtered by $k_{0}(b, p, q)$ and backprojected with a Mojette backprojection, (b) Classical reconstruction with regularly distributed angles filtered by $k_{0}(t, \theta)$ and backprojected with a classical backprojection, (c) Spline 1 reconstruction with uniformly distributed Katz angles filtered by $k_{1}(b, p, q)$ and backprojected with a Mojette backprojection, (d) Classical round image reconstruction with regularly distributed angles filtered by $k_{1}(t, \theta)$ and backprojected with a classical backprojection

(a)

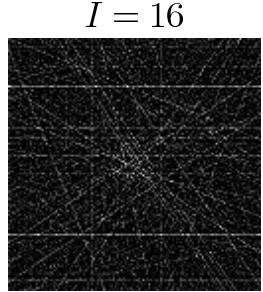

(b)

$\mathrm{MSE}=0.4827$

(c)

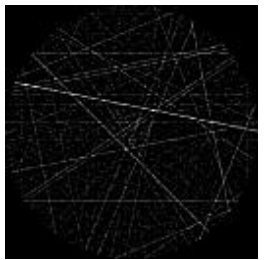

$\mathrm{MSE}=0.5666$

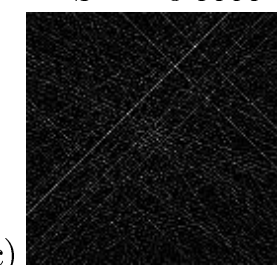

$\mathrm{MSE}=0.5479$

(d)

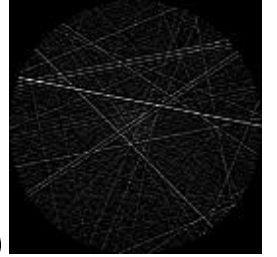

$\mathrm{MSE}=0.5217$
$I=32$

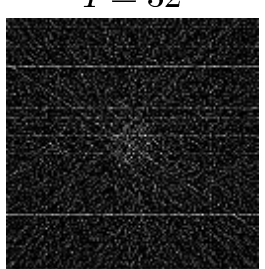

$\mathrm{MSE}=0.4620$

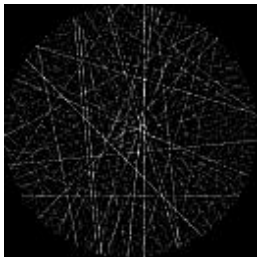

$\mathrm{MSE}=0.5175$

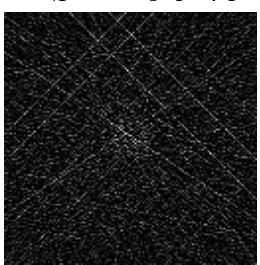

$\mathrm{MSE}=0.4932$

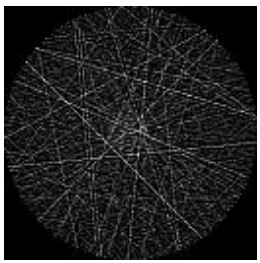

$\mathrm{MSE}=0.4171$

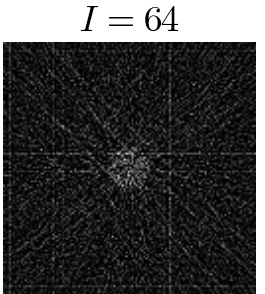

$\mathrm{MSE}=0.4018$

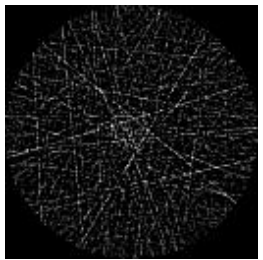

$\mathrm{MSE}=0.4717$

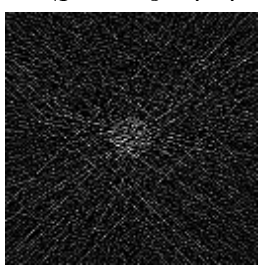

$\mathrm{MSE}=0.4185$

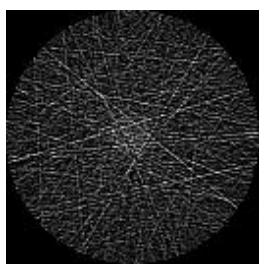

$\mathrm{MSE}=0.3647$

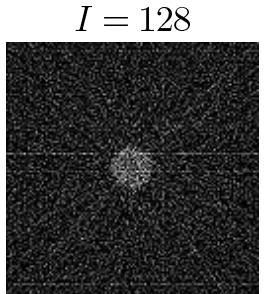

$\mathrm{MSE}=0.3154$

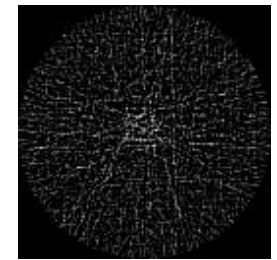

$\mathrm{MSE}=0.4600$

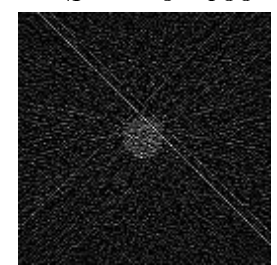

$\mathrm{MSE}=0.4032$

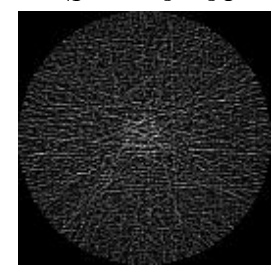

$\mathrm{MSE}=0.3821$

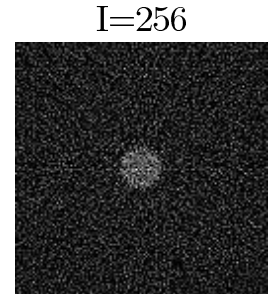

$\mathrm{MSE}=0.3180$

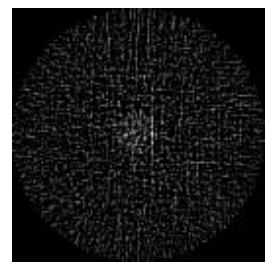

$\mathrm{MSE}=0.4563$

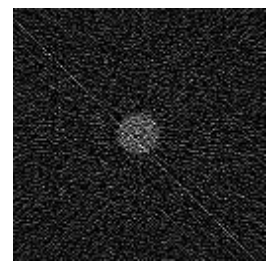

$\mathrm{MSE}=0.3343$

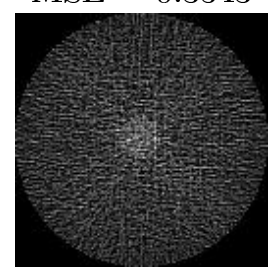

$\mathrm{MSE}=0.2831$ 
only left singularity is for the angle $(p=1, q=1)$ at both locations $b=0$ and $b=p-q$. However, the limit computation from the continuous expression (Eq. 10) of each side of $t$ gives the results. Of course for higher spline order, the differentiability of the spline functions does not produce any trouble. The continuous-discrete equivalence allows for having both the spline coefficients images and their sampled counterpart using the postfilter described in Unser-AlDroubi works and that can be fastly imlemented. The big advantage is to compute the infinite Spline Ramp filter (the costly part of the FBP algortihm) only once and to produce different images thereafter as made for the previous spline implementation. The first visual conclusions for noisy reconstructions of different spline order are the very close behavior betweenspline order images as already found for the classical spline FBP. However, as explained in $^{3}$ when a global image processing as to be done after the reconstruction, the total loss of information will be obtained by staying in the same spline functional subspace.

\subsection{Noise phantoms and the noise distribution among bins}

The simple square and circle phantoms were designed with a non-null background such that we are able to compare the reconstructions with and without Poisson's noise. Square corners are likely to be rare in a medical image (this can however happens with bones) but since this produces high frequencies in the corner of the Fourier domain, this is important to ckeck the LSI (linear shiftinvariantness) property of the algorithm. This property can be seen with and without noise : there are no differences between the kind of artifacts we obtained for the square or the disk.

The second important fact is that the simulated noise has the same structure in the reconstructed image both for standard and Mojette FBP. This is very interesting since the noise distribution was made in a very different fashion to follow the Poisson process since the number of bins per projection and the amount of information into a bin is different in the two methods. In other words, the Mojette reconstruction method that first takes the initial data and maps each bin into the discrete grid is also valid for noisy information. This result was not taken for granted since all the properties of the Mojette operator are projection based whereas the noise only acts on the bin basis. The only link between projection and bin lies in the total amount of information that is kept constant, so for the noise.

The third lesson that we have comes from the way of dispersing the initial noisy information according to the number of projections. Even if the MSE of the center of the reconstruction as well as the visual inspection are not objective measures, it is obvious that the quality of the reconstruction for both standard and Mojette spline reconstructions improves as the number of angles increases. Here again, the specificities of the FBP technique(streak artifact phenomenon) is proeminent over the discrete geometry parameter. Here again this result was quite obvious for noise free data whereas it is not for noisy bins since there are many projections where the number of bins is high so the recorded information is low, resulting into a decrease of the signal to noise ratio per bin.

\subsection{Figure of merit and computable distances}

As already mentioned, the quality of the reconstructions have still to be well established. The direct comparison between standard and Mojette spline 0 FBP reconstructions always gives the same results for all tests. In a way, the mean square error (MSE) of the center of the picture is always lower for the Mojette technique and the visual inspection makes the disk or square more easily detectable for the Mojette versus standard technique. This conjunction allows to think that a filter matchering as perform by an ideal or quasi-ideal observer will give the same result. These observer studies will be conducted now. It consists in two different detection tasks as described in. ${ }^{8}$ The first is the low-contrast detection task between the signal present or absent (either made for the disk or the square). The second is the high-frequency task to distinghish between the square and the disk in a noisy context. 


\section{CONCLUSION}

In this paper, the tomographic reconstruction using the Mojette spline FBP was presented, compared to the standard spline FBP reconstruction and assessed in a noisy context. The standard spline FBP reconstruction was known to respect the properties of the signal and noise as well as the LSI properties of the FBP. The result of this study shows that its Mojette counterpart overcomes these results. This was not surprising from noisy free data because of the mathematical properties of the inverse operator. However, it can be interpreted as a powerful inheritance of the stability of this inverse Mojette operator characteristics that the Mojette spline FBP performs as well in a noisy context. An observer study will confirm these results both for low-contrast and high-frequency tasks.

\section{REFERENCES}

1. Akram Aldroubi and Michael Unser. Sampling procedures in function spaces and asymptotic equivalence with Shannon's sampling theory. Numer. Funct. Anal. and Optimiz., 15:1-21, 1994.

2. Jeanpierre Guédon, Stéphane Beaumont, Myriam Servières, and Nicolas Normand. The 3D Mojette Projector Applied to PET and Radiotherapy Imaging. Proc. of the IEEE ISBI conference, Washington D.C., pages 836-839, April 2004.

3. JeanPierre Guédon and Yves Bizais. Separable and radial bases for medical image processing. SPIE lmage Processing, 1898:652-661, 1993.

4. JeanPierre Guédon and Yves Bizais. Bandlimited and haar filtered back-projection reconstuction. IEEE Transaction on Medical Imaging, 13(3):430-440, September 1994.

5. Myriam Servières, JeanPierre Guédon, and Nicolas Normand. A discrete tomography approach to PET reconstruction. In Yves Bizais, editor, Fully 3D Reconstruction In Radiology and Nuclear Medicine, June 2003.

6. Myriam Servières, Nicolas Normand, Peggy Subirats, and JeanPierre Guédon. Some links between continuous and discrete radon transform. In Proc. SPIE Vol. 5370, Medical Imaging 2004: Image Processing, pages 1961-1971. SPIE, May 2004.

7. Peggy Subirats, Myriam Servières, Nicolas Normand, and JeanPierre Guédon. Angular assessement of the mojette filtered back projection. In Proc. SPIE Vol. 5370, Medical Imaging 2004: Image Processing, pages 1951-1960. SPIE, May 2004.

8. Robert F. Wagner and David G. Brown. Unified SNR analysis of medical imaging systems. Phys. Med. Biol., 30(6):489-518, 1985. 